\title{
A Knife-edge Input Point Spread Function Estimation Method for Document Images
}

\author{
Jianqiang Zhong \\ Teaching and research department \\ High-tech institute, Fan Gong-ting south street T on the $12^{\text {th }}$, Qing Zhou, China \\ 794873071@qq.com
}

\begin{abstract}
In this paper the progress of document image Point Spread Function (PSF) estimation will be presented. At the beginning of the paper, an overview of PSF estimation methods will be introduced and the reason why knife-edge input PSF estimation method is chosen will be explained. Then in the next section, the knife-edge input PSF estimation method will be detailed. After that, a simulation experiment is performed in order to verify the implemented PSF estimation method. Based on the simulation experiment, in next section we propose a procedure that makes automatic PSF estimation possible. A real document image is firstly taken as an example to illustrate the procedure and then be restored with the estimated PSF and Lucy-Richardson deconvolution method, and its OCR accuracy before and after deconvolution will be compared. Finally, we conclude the paper with the outlook for the future work.
\end{abstract}

Keywords-Point Spread Function; document image; Knife-edge Input

\section{INTRODUCTION}

The knife-edge input method is widely used because it is least demanding in terms of image scene content. The method relies on edge structures, which can be easily identified as they frequently occur in document images. Therefore, the knife-edge input method has been applied to document images[1]. In this project we will develop a technical solution for document image PSF estimation based on the knife-edge input method.

\section{KNIFE-EDGE INPUT METHOD}

In this section, firstly we will detail on the theoretical part of this method. Formulation of a set of simulation images to verify the potential of knifeedge input method will be done in the next section.

\subsection{System PSF}

The system PSF of the scanner imaging system consists of several components: the optical PSF $P S F_{\text {opt }}$ accounts for the blurring induced by the optics; the image motion PSF $P S F_{\text {im }}$ is related to detector integration time and scanner movement; the detector PSF PSF $F_{d e t}$ is caused by the non-zero area of each detector in the sensor; the electronics PSF PSF $F_{e l}$ is a low-pass filter used for reducing noise. The system PSF is therefore the convolution of the different component PSFs given by:

$P S F_{\text {sys }}=P S F_{\text {opt }} * P S F_{\text {im }} * P S F_{d e t} * P S F_{\text {el }}$

It is very difficult to determine the exact shape of the system PSF. In this article the system PSF is assumed to be circular Gaussian, determined by only one parameter, namely, the standard derivation of 2D Gaussian function:

$$
\operatorname{PSF}(x, y \mid \delta)=\frac{1}{2 \pi \delta^{2}} e^{-x^{2} / 2 \delta^{2}} e^{-y^{2} / 2 \delta^{2}}
$$

Elisa H. Barney Smith found a more proper choice for scanner system PSF should be Cauchy function rather than Gaussian function [2]. However, because of the good properties of Gaussian functional form, we prefer to use Gaussian and the developed technique can be easily extended to Cauchy function.

The knife-edge input method is based on the fact that a knife-edge will excite the imaging system in all spatial frequencies. The profile of the edge is called the Edge Spread Function (ESF), which is the projection of the 2D PSF along the edge's normal direction. From the ESF a Linear Spread Function (LSF) can be estimated, which is also Gaussian, and which can be determined by one parameter, namely, its standard deviation $\delta_{t}$ :

$$
\operatorname{LSF}\left(t \mid \delta_{t}\right)=\frac{1}{\sqrt{2 \pi} \delta_{t}} e^{-t^{2} / 2 \delta_{t}^{2}}
$$

The standard deviation of the measured LSF $\left(\delta_{t}\right)$ is equal to the standard derivation of the system PSF in the case of circular 2D Gaussian PSF estimation (the case for this project report). In the case where the 2D Gaussian PSF is elliptical multiple of measured LSFs are needed in order to fit the 2D Gaussian PSF parameters. 


\subsection{ESF and PSF Parameter Estimation}

We assume that the edge of the image patch where the ESF is extracted has been identified with the Canny detector. After that the ESF is obtained by projecting every pixel in the edge cross-section onto the edge normal and then calculating its distance to the edge, which is in fact the distance from the pixel position (x, y) to the estimated edge line, with $a$ and $b$ the linear regression parameters describing the edge position:

$$
d(x, y)=\frac{a x-y+b}{\sqrt{a^{2}+1}}
$$

In the case where vertical or horizontal edge lines are identified, the horizontal or vertical coordinate distance between the point $(\mathrm{x}, \mathrm{y})$ to the estimated edge line can be regarded as the distance to the edge. However, from the perspective of ESF estimation, we expect the edge line should have subtle angle disparity with the vertical or horizontal direction as more samples can be used for ESF estimation in this case.

To define the relationship between ESF and LSF, we can have the following formula:

$$
\begin{aligned}
& \operatorname{ESF}(x)=\quad \operatorname{LSF}(x) \otimes U(x) \\
& =\int_{-\infty}^{x} \operatorname{LSF}(t) U(x-t) d t+\int_{x}^{+\infty} \operatorname{LSF}(t) U(x-t) d t \\
& =\quad w \int_{-\infty}^{x} L S F(t) d t+v \int_{x}^{\infty} L S F(t) d t \\
& =w\left(\int_{-\infty}^{\infty} L S F(t) d t-\int_{x}^{\infty} L S F(t) d t\right)+v \int_{x}^{\infty} L S F(t) d t \\
& =\quad w+(v-w) \int_{x}^{\infty} \frac{1}{\sqrt{2 \pi} \delta_{t}} e^{-\frac{(t-m)^{2}}{\delta_{t}^{2}}} d t \\
& =\quad w+(v-w) \frac{1}{\sqrt{\pi}} \int_{\frac{x-m}{\sqrt{2} \delta_{t}}}^{\infty} e^{-t^{2}} d t \\
& =\quad(w)+(v-w) \frac{1}{2} \operatorname{erfc}\left(\frac{x-m}{\sqrt{2} \delta_{t}}\right) \\
& =\quad\left(1-\frac{1}{2} \operatorname{erfc}\left(\frac{x-m}{\sqrt{2} \delta_{t}}\right)\right)(w-v)+v \\
& =\operatorname{ESF}\left(x \mid \delta_{t}, m, w, v\right)
\end{aligned}
$$

where $m$ is a constant, introduced to account for the disturbance of noise and $\operatorname{erfc}()$ is the complementary error function, $w$ and $v$ stand for background and foreground gray value respectively.

Although there are four unknown variables in (5) $\left(\delta_{t}, m, w, v\right)$, in the context of PSF estimation we are only interested in $\delta_{t}$. In this report we are using Gradient descent method.
To sum up, the steps of estimating the ESF parameters are as follows:

1) For each pixel in the edge cross-sections calculate its projection onto the edge normal, and obtain $\mathrm{d}(\mathrm{x}, \mathrm{y})$ according to (4), which corresponds to its $\mathrm{x}$-coordinate. The y-coordinate of the pixel is its pixel grey value.

2) Initialize the unknown variables $\left(\delta_{t}, \mathrm{~m}, \mathrm{w}, \mathrm{v}\right)$ in (5). $\delta_{t}$ is initialized to $1, m$ is set to be the position of the edge line, $w$ and $v$ can be found based on the histogram analysis of image patches on either side of the detected edge: the gray pixel value corresponding to the peak of the histogram is selected as $m$ or $v$.

3) Use all the ESF measurements to estimate the unknown variables with the Nelder-Mead method or gradient descent method to obtain the estimated $\operatorname{ESF}\left(\mathrm{x} \mid \delta_{t}\right.$, $\mathrm{m}, \mathrm{w}, \mathrm{v})$.

4) For each ESF measurement (point in the ESF profile) calculate its model residual $r(x)=y-\operatorname{ESF}(x)$; compute the standard deviation of $\mathrm{r}(\mathrm{x})$ and record it as $w_{r}$, and $w_{r}$ can be regarded as ESF parameter estimation confidence level as low $w_{r}$ indicates that data can fit the estimated function very well and vice versa.

After each ESF measurement is estimated, the PSF parameter estimation is straight-forward for our case: we can calculate either the mean value of $\delta_{t} \mathrm{~s}$ or the weighted mean value based on measured $\delta_{t} \mathrm{~s}$ and $w_{r} \mathrm{~s}$ in step 4) as the final PSF parameter $\delta$. In this project we use the weighted mean method.

\section{AUTOMATIC SELECTION OF EDGE REGIONS FOR PSF ESTIMATION \\ 3.1 Edge region selection}

In order to select long and broad image regions of homogeneous foreground and background with reasonable contrast, we propose the following processing chain:

\section{1) Canny edge detection}

Canny edge detector has been widely used in the field of image processing, and alternative edge detection methods such as Sobel edge detector can also be used in this processing chain.

\section{2) Edge coding}

The purpose of edge coding is to find straight lines from all the detected lines. The algorithm we have adopted is form [3].

\section{3) Filtering of the recoded image}

The purpose of recoded image filtering is to identify the long edge regions, and this step is also well explained in [4]. 
4) Select the edge that has reached the maximum value

In the recoded image maximum gray value pixels represent long and straight edge, which will be selected as candidate edge regions.

\section{5) Edge region size filtering}

This stage can be combined with stage 3 ) and it is a procedure that removes small edge regions.

\section{6) Edge region homogeneity filtering}

The purpose of step is to find the homogeneous edge regions. In order to do that an homogeneity criterion from [4] is adopted:

$$
\frac{E\left\{(x-u)^{2}\right\}^{3}}{E\left\{(x-u)^{4}\right\}}
$$

If the homogeneity criterion is low it means the foreground or background is homogeneous.

\section{7) Edge region contrast filtering}

In this step the contrast between foreground and background is calculated, and if the contrast of the edge region is small then it will be discarded from further analysis.

\section{8) Select the best ESF profile}

For each candidate edge regions potential ESF profile will be evaluated based on a quantitative measurement, and best ESF profile in this edge region will be selected. regions

9) Perform ESF estimation on selected edge

In this step ESF parameter will be estimated with the function fitting scheme we proposed before.

\section{0) ESF parameter estimation evaluation}

After obtaining the ESF parameter we will evaluate the result with the stand derivation of data fitting disparity. Only ESF profile with good data fitting will be used for PSF estimation.

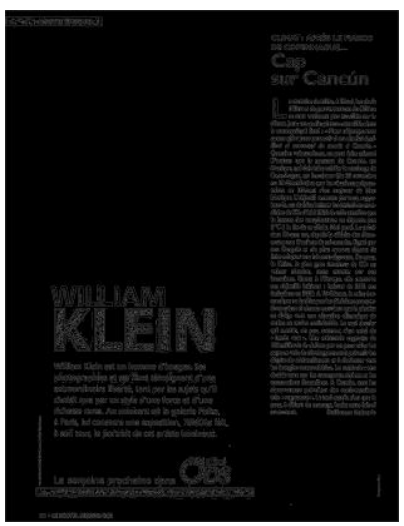

(a)

\section{(b)}

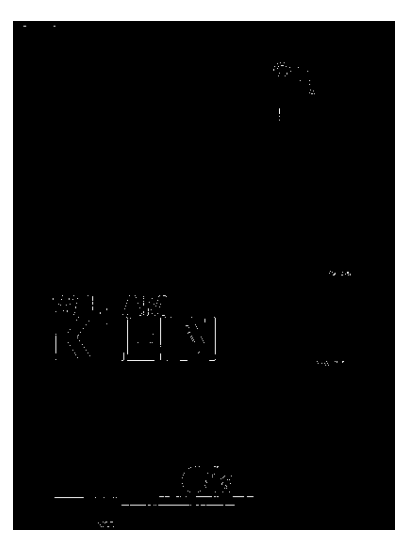

Fig. 2 Comparison of edge maps

\section{1) PSF estimation}

In this step the PSF is calculated with the weighted mean square method.

Some intermediate results will be shown in order to further illustrate the procedure. The example image is shown in Fig.1, which was provided by Adobe. Fig. 2 compares the Canny edge detection image (Step 1) and the filtered encoded image (Step 3 ). As we can see from the comparison only large straight lines are kept as clues of selecting candidate edge regions. Fig. 3(a) shows one selected edge region (red asterisks) as well as the best edge profile location (red circle), and its corresponding ESF as well as the estimated function are shown in Fig. 3(b). Fig. 4 illustrates all the detected edge regions for PSF estimation, and in total 21 edge regions are selected, and careful examination shows that most of the edge regions are located around large characters or homogenous regions in the pictorial part of the document image, which is a reasonable choice when people select edge regions for PSF estimation manually. Based on the estimated ESF, the PSF can be also calculated, which is 1.2148 .

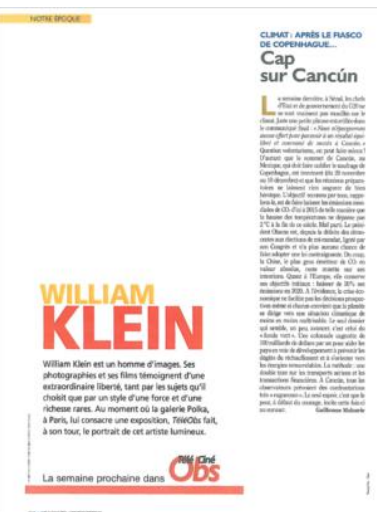

Fig. 1 Example image for PSF estimation

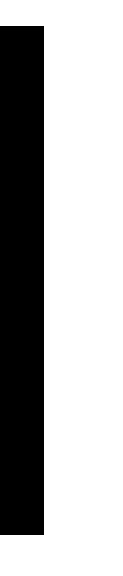

$$
\text { . }
$$




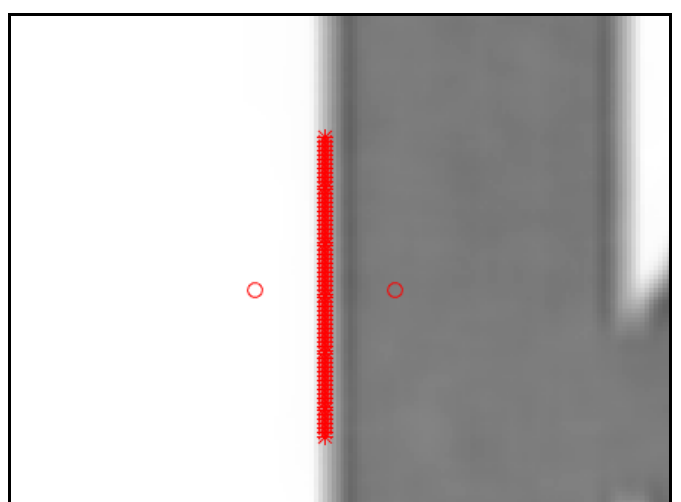

(a)

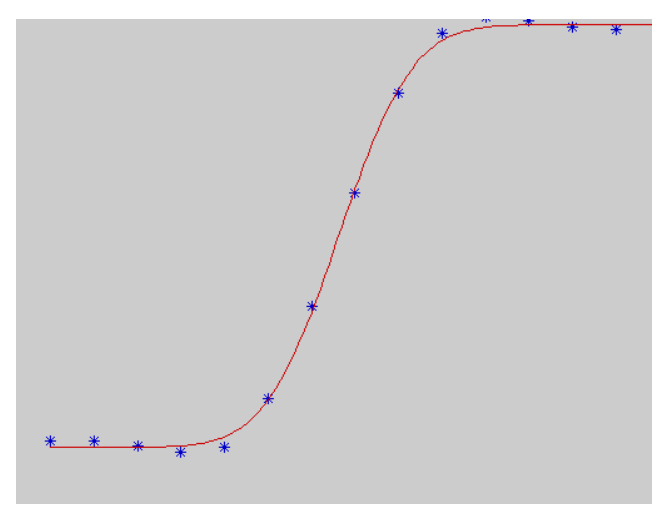

(b)

Fig. 3 (a) Selected edge region and best ESF location ; (b) ESF profile as well as its underlying function.

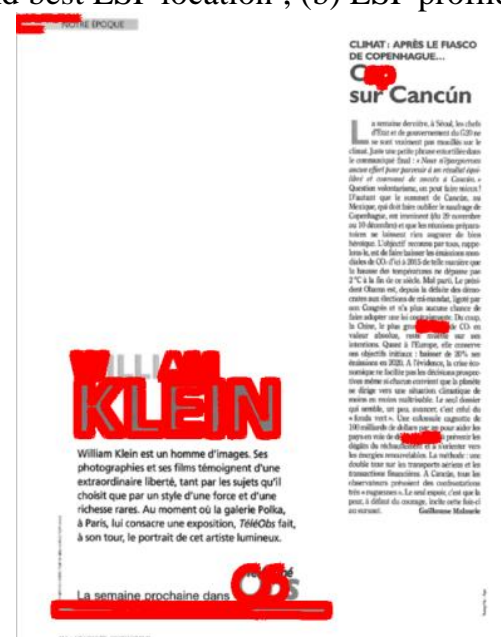

Fig. 4 Selected edge regions

\subsection{Evaluate the estimated PSF with OCR accuracy}

In order to evaluate the estimated PSF for the example image, image deconvolution is performed. After that we perform OCR with the open-source engine Tesseract [5], and compare the OCR

\section{William Klein est un homme d'images. Ses photographies et ses films témoignent d'une extraordinaire liberté, tant par les sujets qu'il choisit que par un style d'une force et d'une richesse rares. Au moment où la galerie Polka, à Paris, lui consacre une exposition، TéléObs fait, à son tour, le portrait de cet artiste lumineux.}

(a) accuracy difference before and after image deconvolution with the estimated PSF parameter.

Fig. 5 (a) shows the extracted text image region before deconvolution, and after deconvolution the text image patch is shown in Fig. 5 (b). It is obvious that after image deconvolution its quality has improved.

\section{William Klein est un homme d'images. Ses photographies et ses films témoignent d'une extraordinaire liberté, tant par les sujets qu'il choisit que par un style d'une force et d'une richesse rares. Au moment où la galerie Polka, à Paris, lui consacre une exposition, TéléObs fait, à son tour, le portrait de cet artiste lumineux.}

(b)

Fig. 5 (a) Original image (b) After image deconvolution

OCR accuracy comparison is shown in Table I, and from table we can see that image deconvolution with our automatically selected PSF can improve OCR accuracy. We also compare the deconvolution result with the manually selected PSF, which was done in our last project report. From this example we can see automatically selected PSF and manually selected PSF can reach similar OCR accuracy. 
Table I Comparison of OCR accuracy before and after image deconvolution

\begin{tabular}{|c|c|}
\hline Method & OCR Accuracy \\
\hline Without deconvolution & $83.27 \%$ \\
\hline Manually selected PSF for deconvolution & $90.81 \%$ \\
\hline $\begin{array}{c}\text { Automatically selected PSF with our proposed method for } \\
\text { deconvolution }\end{array}$ & $92.26 \%$ \\
\hline
\end{tabular}

\section{CONCLUSION}

The prelimilary PSF estimation research gives very promising results for future research. First of all, we have implemented all the important technical components (ESF function fitting, ESF profile extraction, homogeneous criteria and so on ) in the PSF estimation processing chain. When PSF estimation is finally applied to the real document image data, an automatic procedure is also proposed.

\section{Reference}

[1] Y. Tian, W. Ming: Adaptive deblurring for camera- based document image processing. Lecture Notes in Computer Science, 5876 (2009), 767-777 (Konica Minolta Technology Report, 8 (2011), 105- 110)
[2] E.H. Barney-Smith, "PSF estimation by gradient descent fit to the ESF", SPIE Image Quality and System Performance III, vol. 6059, 2006

[3] O.Shacham, , O. Haik \& Y. Yitzhaky, Blind restoration of atmospherically degraded images by automatic best step-edge detection. Pattern Recognition Letters, November, 28(15), 2007, pp.2094-2103

[4]

Shacham, , O. Haik \& Y. Yitzhaky, Blind restoration of atmospherically degraded images by automatic best step-edge detection. Pattern Recognition Letters, November, 28(15), 2007, pp.2094-2103

[5] https://code.google.com/p/tesseract-ocr/

[6] http://en.wikipedia.org/wiki/Richardson\%E 2\%80\%93Lucy_deconvolution 